\title{
SIGNAL STRENGTH LOCALIZATION BOUNDS IN AD HOC \& SENSOR NETWORKS WHEN TRANSMIT POWERS ARE RANDOM
}

\author{
Neal Patwari and Alfred O. Hero III \\ Department of Electrical Engineering \& Computer Science \\ University of Michigan, Ann Arbor, USA \\ [npatwari, hero]@eecs.umich.edu
}

\begin{abstract}
Sensor localization bounds have been derived assuming that received signal strength (RSS) measurements are performed with perfectly known sensor transmit powers. In this paper the Bayesian Cramér-Rao bound is derived assuming transmit powers are random with known prior distribution. Further, both directional measurements on each link, from $i$ to $j$ and from $j$ to $i$, and their correlation, are explicitly considered. Results show that random transmit powers have a small (5-13\%) impact on coordinate estimation bounds. However, using only the average of the directional measurements can significantly increase these bounds.
\end{abstract}

\section{INTRODUCTION}

As part of the self-organizing nature of wireless sensor networks, sensors will perform cooperative localization to estimate their own coordinates. In cooperative localization, a small fraction of nodes will have a priori known coordinates, either because they are fixed and have been given their coordinate by a human moderator, or because they are capable of estimating their coordinate using an independent method, such as GPS. This paper addresses the case in which sensors measure the received signal strength (RSS) of messages sent by nearby neighbors in the network. The RSS measurements and prior coordinate information are used to estimate the coordinates of all sensors. Measurement of RSS also plays an important part in acoustic and RF source localization in networks of asynchronous sensors.

A system designer bears the burden of ensuring that the accuracy of the coordinate estimates will meet the requirements of the application. Applications such as geographicbased routing, inventory management and logistics, environmental monitoring, and source localization, will all require sensor localization, at certain degrees of accuracy.

The Cramér-Rao bound (CRB) $[1,2,3,4,5]$ and the Bayesian CRB [6] have been used to provide a tool to aid in

This work was partially funded by the DARPA Defense Sciences Office under Office of Naval Research contract \#N00014-04-C-0437. Distribution Statement A. Approved for public release; distribution is unlimited. the design of cooperative localization systems. Such bounds help us know what accuracies cannot be achieved by a sensor network with a particular design. This paper extends the analysis of cooperative localization from RSS measurements in two aspects:

1. Both directional measurements (from $i$ to $j$, and from $j$ to $i$ ) and their correlation are considered explicitly,

2. The transmit power is not assumed to be perfectly known.

Both represent realistic representations of RSS measurements in wireless sensor networks. Item (1.) helps quantify the benefit in measuring RSS in both directions of a link between two sensors. Item (2.) helps to quantify the loss in accuracy when the transmit powers of each sensor is not known exactly. In realistic sensor networks, due to the cost of device calibration, sensors do not know their precise transmit power level. While they may report that they are transmitting at a nominal power level, their actual transmit power may vary by a few $\mathrm{dB}$ because of device manufacturing variations, because of battery level, or because of the position of and objects near the antenna.

Further, joint source and sensor localization bounds will benefit from these extensions. In passive source localization, a sensor measures the RSS of a source signal whose source energy is largely unknown.

\section{PROBLEM STATEMENT AND MODELS}

The cooperative localization problem is the estimation of sensor coordinates $\mathbf{z}_{i}=\left[x_{i}, y_{i}\right]^{T}$ for sensors $i=1 \ldots n$ (where ${ }^{T}$ indicates transpose), given the a priori known coordinates $\mathbf{z}_{i}$ for $i=n+1 \ldots N$, where $N=n+m$. This paper assumes $2 \mathrm{D}$ coordinates, although the analysis could be readily extended to the $3 \mathrm{D}$ case.

It is assumed that measurements of received signal strength are made between many pairs of sensors. In particular, $P_{i, j}$, the received signal strength measured at $i$ of the signal transmitted by $j$, is measured for each pair $(i, j)$ in a measure- 
ment set $H$. This set $H$ is a subset of $\{1, \ldots, N\}^{2}$, since not all pairs may be in range, or limited bandwidth may preclude full measurements. Also, $(i, i)$ is not in $H$ since self-RSS measurement is not done.

\subsection{Pair Measurement Correlation}

Two measurements can be made between each pair of sensors $(i, j)$ : one with $i$ as the transmitter and $j$ as the receiver, and the other with them switched. The measurements tend to be very correlated, due to the multipath shadowing channel which affects both directional links identically. Past research has assumed, as a simplification, that the two measurements were perfectly correlated [2].

This paper extends the analysis by considering that the two directional measurements are not perfectly correlated. These differences come from: additive noise, movement in the channel in the time between the two measurements, and differences in the transmitter and receiver characteristics of the two devices. We assume that $P_{i, j}$ and $P_{j, i}$ are correlated with coefficient $0 \leq \rho \leq 1$, as specified in Section 2.3. We also consider in Section 4.1.3 the effect of estimating coordinates using only the average, $\frac{1}{2}\left(P_{i, j}+P_{j, i}\right), \forall i, j$.

\subsection{Random Transmit Power}

This paper also avoids the assumption that the transmit power is known. In particular, we consider $\Pi_{0 i}$, the signal power $(\mathrm{dBm})$ at a distance of $\Delta_{0}=1 \mathrm{~m}$ from the antenna of sensor $i$. By considering the power slightly away from the transmitter antenna, we effectively consider both the device and battery variation and the variation in the antenna and the environment immediately around the antenna. In an idealized environment, $\Pi_{0 i}$ is given by the Friis transmission equation, and in a real channel, the near-field environment affects the antenna impedance and thus the antenna radiation efficiency [7].

We also assume that $\Pi_{0 i}$ is constant across many measurements. While battery levels and the near-field environment may change over time, we assume that these effects are constant during short periods of RSS measurements.

\subsection{Combined Channel Model}

In particular, we model the vector $\mathbf{p}_{i j}=\left[P_{i, j}, P_{j, i}\right]^{T}$ as bivariate Gaussian with mean $\boldsymbol{\mu}_{i j}$ and covariance $C_{i j}$, where

$$
\begin{aligned}
\boldsymbol{\mu}_{i j} & =\left[\begin{array}{c}
\Pi_{0 j}-10 n_{p} \log _{10} \frac{\left\|\mathbf{z}_{i}-\mathbf{z}_{j}\right\|^{2}}{\Delta_{0}^{2}} \\
\Pi_{0 i}-10 n_{p} \log _{10} \frac{\left\|\mathbf{z}_{i}-\mathbf{z}_{j}\right\|^{2}}{\Delta_{0}^{2}}
\end{array}\right], \\
C_{i j} & =\sigma_{d B}^{2}\left[\begin{array}{ll}
1 & \rho \\
\rho & 1
\end{array}\right],
\end{aligned}
$$

where $n_{p}$ is the path loss exponent of the environment of interest, and $\rho$ is the correlation coefficient, $0 \leq \rho \leq 1$. To provide an intuitive feel for practical values of the parameters in (1), from the RSS measurements reported in [2], we can calculate that $\sigma_{d B}=4.25 \mathrm{~dB}, n_{p}=2.30$, and $\rho=0.704$. We further assume that $\left\{\mathbf{p}_{i j}\right\}_{i, j}$ are statistically independent. The independence assumption is for simplification; future work should address the effects of correlations between different links in the network.

\section{BOUNDS ANALYSIS}

The unknown parameters defined in Section 2 are $\boldsymbol{\theta}$,

$$
\begin{aligned}
\boldsymbol{\theta} & =\left[\mathbf{x}^{T}, \mathbf{y}^{T}, \boldsymbol{\Pi}^{T}\right]^{T} \\
\mathbf{x} & =\left[x_{1}, \ldots, x_{n}\right]^{T} \\
\mathbf{y} & =\left[y_{1}, \ldots, y_{n}\right]^{T} \\
\mathbf{\Pi} & =\left[\Pi_{01}, \ldots, \Pi_{0 N}\right]^{T}
\end{aligned}
$$

There are $2 n+N$ parameters since none of the $N$ sensors have perfect knowledge of their transmit power, and $n$ of the sensors have no prior coordinate knowledge. We assume perfect prior coordinate information for sensors $n+1$ through $N$, and an i.i.d. Gaussian prior on $\Pi_{0 i}$ for all sensors $i$, with variance $\sigma_{\Pi}^{2}$ and mean $\bar{\Pi}_{0}$.

The Bayesian Cramér-Rao Bound [8, pp. 72-73] (also called the van Trees inequality or posterior CRB) states that any estimator, $\hat{\boldsymbol{\theta}}$, must have error correlation matrix $R_{\epsilon}$ satisfying

$$
\begin{aligned}
R_{\epsilon} & \geq \mathbf{F}^{-1} \triangleq\left[\mathbf{F}_{\boldsymbol{\theta}}+\mathbf{F}_{P}\right]^{-1}, \\
\text { where } R_{\epsilon} & \triangleq \mathrm{E}\left[(\hat{\boldsymbol{\theta}}-\boldsymbol{\theta})(\hat{\boldsymbol{\theta}}-\boldsymbol{\theta})^{T}\right],
\end{aligned}
$$

$\mathbf{F}_{\boldsymbol{\theta}}$ is the Fisher information matrix, and $\mathbf{F}_{P}$ is the prior information matrix,

$$
\begin{aligned}
\mathbf{F}_{\boldsymbol{\theta}} & =-\mathrm{E}\left[\nabla_{\boldsymbol{\theta}}\left(\nabla_{\boldsymbol{\theta}} \ln f\left(\left\{\mathbf{p}_{i j}\right\}_{i, j} \mid \boldsymbol{\theta}\right)\right)^{T}\right], \\
\mathbf{F}_{P} & =-\mathrm{E}\left[\nabla_{\boldsymbol{\theta}}\left(\nabla_{\boldsymbol{\theta}} \ln f(\boldsymbol{\theta})\right)^{T}\right],
\end{aligned}
$$

where $\mathrm{E}[\cdot]$ indicates expected value, and $\nabla_{\boldsymbol{\theta}}$ is the gradient operator w.r.t. $\boldsymbol{\theta}$.

\subsection{Average and Difference of Measurement Pairs}

Before calculating the Fisher information, we transform the two directional measurements $\mathbf{p}_{i j}$ for the purposes of discussion. It can be seen both intuitively and from (11) that a full-rank transformation of the measurements does not change their Fisher information. We choose to transform each pair of measurements $\mathbf{p}_{i j}$ by orthogonal matrix $A$,

$$
\tilde{\mathbf{p}}_{i j}=A \mathbf{p}_{i j}, \quad A=\frac{1}{2}\left[\begin{array}{cc}
1 & 1 \\
1 & -1
\end{array}\right] .
$$

For notational purposes, we denote $\tilde{\mathbf{p}}_{i j}=\left[\bar{p}_{i j}, p_{i j}^{\Delta}\right]^{T}$. The top element $\bar{p}_{i j}$ is the average of the two measurements $P_{i, j}$ 
and $P_{j, i}$, and the bottom element, $p_{i j}^{\Delta}$ is half of their difference. These two random variables are still Gaussian, but they are independent, since

$$
\tilde{R}_{i j}=A^{T} C_{i j} A=\frac{\sigma_{d B}^{2}}{2}\left[\begin{array}{cc}
1+\rho & 0 \\
0 & 1-\rho
\end{array}\right] .
$$

where $\rho$ is the correlation coefficient from (1). Because of (6), $\bar{p}_{i j}$ and $p_{i j}^{\Delta}$ have means $\bar{\mu}_{i j}$ and $\mu_{i j}^{\Delta}$, respectively, where

$$
\begin{aligned}
\bar{\mu}_{i j} & =\frac{\Pi_{0 j}+\Pi_{0 i}}{2}-10 n_{p} \log _{10} \frac{\left\|\mathbf{z}_{i}-\mathbf{z}_{j}\right\|^{2}}{\Delta_{0}^{2}} \\
\mu_{i j}^{\Delta} & =\frac{1}{2}\left(\Pi_{0 j}-\Pi_{0 i}\right) .
\end{aligned}
$$

We define $\overline{\boldsymbol{\mu}}$ and $\boldsymbol{\mu}^{\Delta}$ as the mean for all measured pairs,

$$
\begin{aligned}
\overline{\boldsymbol{\mu}} & =\left[\bar{\mu}_{i_{1} j_{1}}, \ldots, \bar{\mu}_{i_{S} j_{S}}\right]^{T} \\
\boldsymbol{\mu}^{\Delta} & =\left[\boldsymbol{\mu}_{i_{1} j_{1}}^{\Delta}, \ldots, \boldsymbol{\mu}_{i_{S} j_{S}}^{\Delta}\right]^{T}
\end{aligned}
$$

where $i_{1} j_{1}, \ldots, i_{S} j_{S}$ is a listing of each unique pair which makes measurements, and $S$ is the number of unique pairs measured (taken from set $H$ defined in Section 2).

\subsection{Derivation of Fisher Information}

As a result of their independence, we split the Fisher information into two matrices, $\overline{\mathbf{F}}_{\boldsymbol{\theta}}$ for the average measurements $\left\{\bar{p}_{i j}\right\}$, and $\mathbf{F}_{\boldsymbol{\theta}}^{\Delta}$ for the difference measurements $\left\{p_{i j}^{\Delta}\right\}$,

$$
\mathbf{F}_{\boldsymbol{\theta}}=\mathbf{F}_{\boldsymbol{\theta}}^{\Delta}+\overline{\mathbf{F}}_{\boldsymbol{\theta}}
$$

We know that for a vector of multivariate Gaussian measurements with mean $\boldsymbol{\mu}(\boldsymbol{\theta})$ and covariance $C$ (for $C$ not a function of $\boldsymbol{\theta}$ ), that the Fisher information matrix is given by [9],

$$
\mathbf{F}_{\boldsymbol{\theta}}=\left[\nabla_{\boldsymbol{\theta}} \mu(\boldsymbol{\theta})\right]^{T} C^{-1}\left[\nabla_{\boldsymbol{\theta}} \mu(\boldsymbol{\theta})\right]
$$

Thus, for the two terms of (10),

$$
\begin{aligned}
\overline{\mathbf{F}}_{\boldsymbol{\theta}} & =\left[\nabla_{\boldsymbol{\theta}} \overline{\boldsymbol{\mu}}\right]^{T} \bar{C}^{-1}\left[\nabla_{\boldsymbol{\theta}} \overline{\boldsymbol{\mu}}\right] \\
\mathbf{F}_{\boldsymbol{\theta}}^{\Delta} & =\left[\nabla_{\boldsymbol{\theta}} \boldsymbol{\mu}^{\Delta}\right]^{T}\left(C^{\Delta}\right)^{-1}\left[\nabla_{\boldsymbol{\theta}} \boldsymbol{\mu}^{\Delta}\right]
\end{aligned}
$$

where $\overline{\boldsymbol{\mu}}$ and $\boldsymbol{\mu}^{\Delta}$ are from (9) and the covariances are,

$$
\bar{C}=\frac{(1+\rho) \sigma_{d B}^{2}}{2} \mathrm{I}_{2 n+N}, \quad C^{\Delta}=\frac{(1-\rho) \sigma_{d B}^{2}}{2} \mathrm{I}_{2 n+N},
$$

and $\mathrm{I}_{2 n+N}$ is the $2 n+N \times 2 n+N$ identity matrix. The elements of $\nabla_{\boldsymbol{\theta}} \bar{\mu}$ are given as,

$$
\begin{aligned}
& \frac{\partial \bar{\mu}_{i j}}{\partial x_{k}}= \begin{cases}-\alpha\left(x_{k}-x_{j}\right) /\left\|\mathbf{z}_{k}-\mathbf{z}_{j}\right\|^{2}, & \text { if } k=i \\
-\alpha\left(x_{k}-x_{i}\right) /\left\|\mathbf{z}_{k}-\mathbf{z}_{i}\right\|^{2}, & \text { if } k=j \\
0, & \text { otherwise }\end{cases} \\
& \frac{\partial \bar{\mu}_{i j}}{\partial y_{k}}= \begin{cases}-\alpha\left(y_{k}-y_{j}\right) /\left\|\mathbf{z}_{k}-\mathbf{z}_{j}\right\|^{2}, & \text { if } k=i \\
-\alpha\left(y_{k}-y_{i}\right) /\left\|\mathbf{z}_{k}-\mathbf{z}_{i}\right\|^{2}, & \text { if } k=j \\
0, & \text { otherwise }\end{cases}
\end{aligned}
$$

$$
\frac{\partial \bar{\mu}_{i j}}{\partial \Pi_{0 k}}= \begin{cases}1 / 2, & \text { if } k=i \\ 1 / 2, & \text { if } k=j \\ 0, & \text { otherwise }\end{cases}
$$

where $\alpha=10 n_{p} /(\log 10)$. Basically, there are six non-zero elements of $\nabla_{\boldsymbol{\theta}} \bar{\mu}_{i j}$ for each pair $(i, j)$.

For $\nabla_{\boldsymbol{\theta}} \mu^{\Delta}$, we have simply that $\frac{\partial \mu_{i j}^{\Delta}}{\partial x_{k}}=\frac{\partial \mu_{i j}^{\Delta}}{\partial y_{k}}=0$, $\forall i, j, k$, and that

$$
\frac{\partial \mu_{i j}^{\Delta}}{\partial \Pi_{0 k}}= \begin{cases}-1 / 2, & \text { if } k=i \\ 1 / 2, & \text { if } k=j \\ 0, & \text { otherwise }\end{cases}
$$

\subsection{Prior Information}

As stated at the start of this section, we assume an i.i.d. Gaussian prior on $\Pi_{0 i}$ for all sensors $i$, with variance $\sigma_{\Pi}^{2}$ and mean $\bar{\Pi}_{0}$. We assume no prior exists for the coordinates of sensors 1 through $n$, although this analysis could be readily modified to include such effects, as reported in [6]. Because of these assumptions, the prior information matrix $\mathbf{F}_{P}$ is,

$$
\mathbf{F}_{P}=\operatorname{diag}\left\{\left[\mathbf{0}_{n}^{T}, \mathbf{0}_{n}^{T}, \mathbf{1}_{N}^{T} / \sigma_{\Pi}^{2}\right]\right\}
$$

where $\mathbf{0}_{n}$ is an $n$-length vector of zeros, and $\mathbf{1}_{N}$ is an $N$ length vector of ones.

\section{NUMERICAL RESULTS}

Given the analysis of Section 2, in this section, we compute the Bayesian CRB $\mathbf{F}^{-1}$ from (3) for a number of examples. In particular, we summarize each lower bound example by reporting the lower bound on RMS localization error $\bar{\sigma}_{\mathbf{z}}$ and RMS transmit power estimation error $\bar{\sigma}_{\Pi}$,

$$
\bar{\sigma}_{\mathbf{z}}=\frac{1}{n} \operatorname{tr} \mathbf{F}_{\mathbf{z}}^{-1}, \quad \bar{\sigma}_{\Pi}=\frac{1}{N} \operatorname{tr} \mathbf{F}_{\Pi}^{-1}
$$

where $\mathbf{F}_{\mathbf{z}}^{-1}$ and $\mathbf{F}_{\Pi}^{-1}$ are defined as the upper left $2 n \times 2 n$ and the lower right $N \times N$ submatrices of $\mathbf{F}^{-1}$, respectively.

\subsection{Deployment Examples}

In the following three examples, plotted in Fig. 1, we calculate the bound for different sensor network geometries. We plot in the figures the lower bound on the 1- $\sigma$ uncertainty ellipse for $\hat{\mathbf{z}}_{i}$, the estimate of the $i$ th sensor coordinate, for each sensor $i=1 \ldots n$. In each example, we use the parameters $\sigma_{d B}=4.25, n_{p}=2.30$, and $\rho=0.704$, which were calculated using the measurement data set reported in Section IV of [2]. Also in each example, we use the a radius of connectivity $r=5 \mathrm{~m}$, to determine which sensors make measurements. A pair $(i, j)$ is in the measurement set $H$ if and only if $\left\|\mathbf{z}_{i}-\mathbf{z}_{j}\right\|<r$. This use of $r$ is an easy way to represent a realistic (not fully-connected) connection graph 


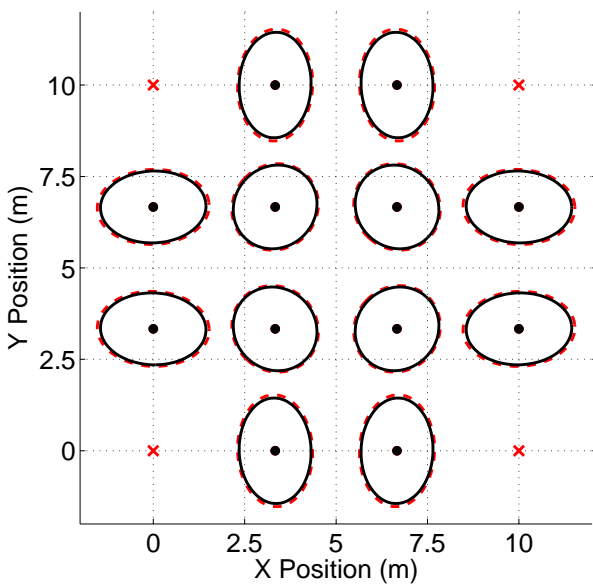

(a)

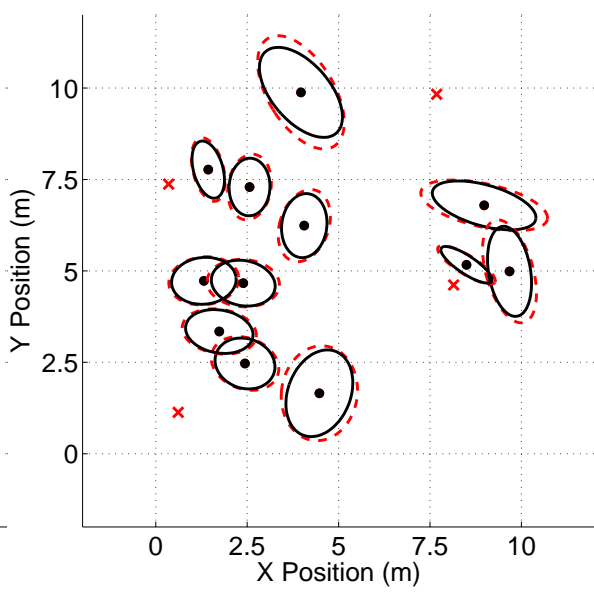

(b)

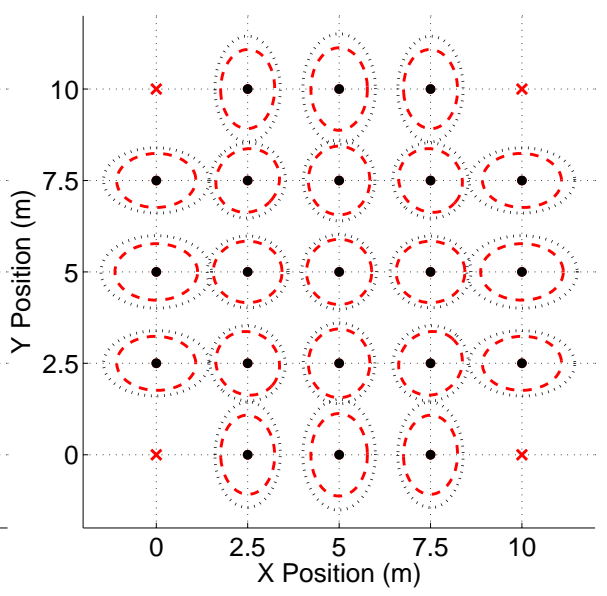

(c)

Fig. 1. Comparison of Bayesian CRB on 1- $\sigma$ uncertainty ellipses when transmit powers are perfectly known (- - ) or random with $\sigma_{\Pi}=5 \mathrm{~dB}(---)$, with unknown (•) and known (x) sensor coordinates shown, for a (a) $4 \times 4$ grid network, (b) random deployment with $N=16$, and (c) $5 \times 5$ grid showing case when $\left\{p_{i j}^{\Delta}\right\}_{i, j}$ are ignored $(\cdots \cdots)$.

for a multi-hop network. Finally, in these three examples, the prior standard deviation $\sigma_{\Pi}$ is set at $5 \mathrm{~dB}$. In the first two examples, we also show the results for the case of perfect prior knowledge of transmit powers, i.e., $\sigma_{\Pi}=0 \mathrm{~dB}$.

We also use a $10 \mathrm{~m}$ by $10 \mathrm{~m}$ square deployment area in each example. These bound results scale with the size of the deployment area and $r$-double the length of each side and $r$, and the standard deviation bound will double. Thus, these results can be extrapolated to an arbitrarily sized area.

\subsubsection{Grid Geometry}

For this example, we test a $4 \times 4$ grid of sensors, spaced regularly across the $10 \mathrm{~m}$ by $10 \mathrm{~m}$ area. The results are shown in Fig. 1(a). If the transmit powers were known exactly, the lower bound on the 1- $\sigma$ uncertainty ellipses would be as shown in Fig. 1(a) by the solid lines (-), and $\bar{\sigma}_{\mathbf{z}}=1.71 \mathrm{~m}$. In the case in which transmit powers are random, the lower bound on the 1- $\sigma$ uncertainty ellipses increases slightly, as shown in Fig. 1(a) by the dashed lines $(----)$, and $\bar{\sigma}_{\mathbf{z}}$ has increased by $4.8 \%$ to $1.79 \mathrm{~m}$. Note that $\bar{\sigma}_{\Pi}=1.58 \mathrm{~dB}$, thus, actual powers can be estimated with considerably more accuracy than $\sigma_{\Pi}$.

\subsubsection{Random Deployment}

Instead of the grid geometry, we now test an $N=16$ sensor network in which sensor coordinates were chosen from a uniform distribution on $[0,10]^{2}$. The $m=4$ sensors closest to the corners of the square are chosen to have knownlocation. All other parameters are kept the same as in the previous example, and the results are shown in Fig. 1(b). Here, for the cases of known and random transmit power, $\bar{\sigma}_{\mathbf{z}}=1.22 \mathrm{~m}$ and $1.37 \mathrm{~m}$, respectively, a difference of $13 \%$.

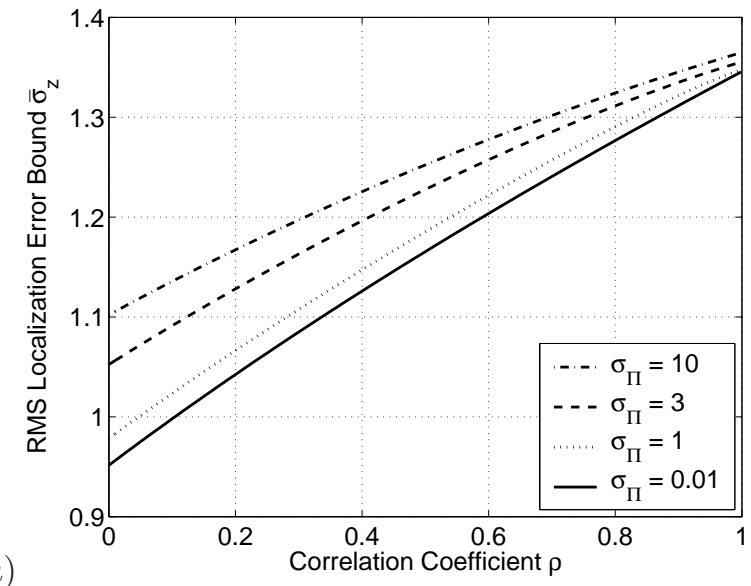

(a)

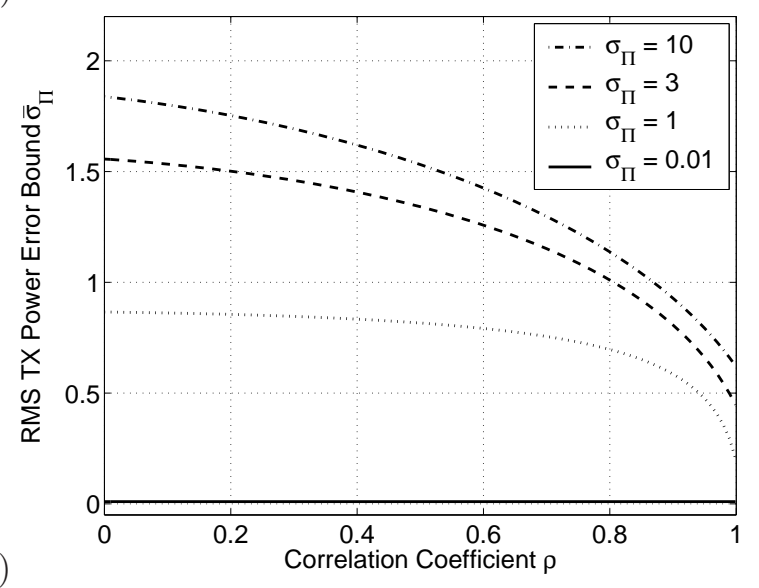

Fig. 2. Bayesian CRB bounds for (a) $\bar{\sigma}_{\mathbf{z}}$ and (b) $\bar{\sigma}_{\Pi}$, for the $5 \times 5$ grid example for four values of $\sigma_{\Pi}$ and a range of $\rho$. 
Typically, for random deployments, we see that the locally higher densities of sensors helps to reduce the lower bound for most sensors. However, with limited range, other random deployments may run into situations in which the leastconnected sensor might make no measurements, or the network as a whole will be disconnected, and as a result, there may not be a finite lower bound. The radius $r$ for randomly deployed sensors may need to be higher to avoid such cases.

\subsubsection{Using Only the Averages $\left\{\bar{p}_{i j}\right\}$}

It may seem natural to use only the link average RSS $\bar{p}_{i j}$ and to discard $p_{i j}^{\Delta}$ in order to reduce by half the measured data in the sensor network. We analyze the effects in this section by replacing (10) with $\mathbf{F}_{\boldsymbol{\theta}}=\overline{\mathbf{F}}_{\boldsymbol{\theta}}$, eliminating the information contained within the measurements $\left\{p_{i j}^{\Delta}\right\}_{i, j}$.

In this example, we use a $5 \times 5$ grid of devices. Other parameters are kept as in the previous examples. The results, as shown in Fig 1(c), show the lower bound for the case when only link averages are considered $(\cdots \cdots)$, and we have that $\bar{\sigma}_{\mathbf{z}}=1.63$. Compared to the case when all information is used, $\bar{\sigma}_{\mathbf{z}}$ has increased $25 \%$ - for the case when all measurements are used (- - -), $\bar{\sigma}_{\mathbf{z}}=1.30$. In particular, the nodes at the edge of the network see their lower bounds expand dramatically along their major axis.

Note that $\bar{\sigma}_{\Pi}=3.40$ in the average-only case, compared to $\bar{\sigma}_{\Pi}=1.24$ in the all-information case. When difference measurements are ignored, it becomes difficult to estimate transmit power. As a result, there is less information about the size or extent of the network, and nodes at the edges are affected most strongly by this lack of knowledge.

\subsection{Performance as a Function of $\rho$ and $\sigma_{\Pi}$}

Finally, we calculate the bound over a range of correlation coefficient $\rho$ and standard deviation of transmit powers $\sigma_{\Pi}$. We use the $5 \times 5$ grid of sensors, with the $m=4$ corner sensors having known-location. The parameters $\sigma_{d B}=4.25$, $n_{p}=2.30$, and $r=5 \mathrm{~m}$ are constant through these calculations. We plot $\bar{\sigma}_{\mathbf{z}}$ in Fig 2(a) and $\bar{\sigma}_{\Pi}$ in Fig 2(b).

Note that as $\rho \rightarrow 1$, the measurement $p_{i j}^{\Delta}$ becomes exactly the difference $\left(\Pi_{0 j}-\Pi_{0 i}\right) / 2$. Since the differences are known perfectly in this case, the only unknown is the absolute, which represents only one degree of freedom. As a result, lower bounds (for localization or transmit power estimation) are not strongly impacted in this case.

Another limiting case is for $\sigma_{\Pi} \rightarrow \infty$. Although it is not shown explicitly, the $\sigma_{\Pi}=10 \mathrm{~dB}$ case in Fig 2 approximates the asymptotic bound very well. As the prior information on $\left\{\Pi_{0 i}\right\}_{i}$ goes to zero, their estimation remains possible, because each sensor has redundancy in the form of many neighbors measuring the RSS of its transmissions.

\section{CONCLUSION}

The Bayesian CRB presents a means to bound the performance of sensor location estimation when two real-world factors are present in the measurements: correlation between the two measurements made on one link, and random sensor transmit powers. This paper presents this bound and compares it to the bound for the ideal case. These factors, for several numerical examples, are shown to increase the lower bound from 5-13\%. In particular, when transmit powers are random, the bound shows the importance of preserving RSS measurements for both directions on each link, instead of summarizing them by their average.

Future work will apply this bound to the joint source and sensor localization problem. It is also possible to derive the lower bound explicitly for the case when $\sigma_{\Pi} \rightarrow \infty$. Finally, it will be critical to develop algorithms robust to random sensor transmit powers, and to test them against these bounds.

\section{REFERENCES}

[1] R. L. Moses, D. Krishnamurthy, and R. Patterson, "A selflocalization method for wireless sensor networks," EURASIP Journal on Applied Sig. Proc., no. 4, pp. 348-358, Mar. 2003.

[2] N. Patwari, A. O. Hero III, M. Perkins, N. Correal, and R. J. O'Dea, "Relative location estimation in wireless sensor networks," IEEE Trans. Signal Process., vol. 51, no. 8, pp. 21372148, Aug. 2003.

[3] N. Patwari and A. O. Hero III, "Using proximity and quantized RSS for sensor localization in wireless networks," in 2nd ACM Workshop on Wireless Sensor Networks \& Applications (WSNA'03), Sept. 2003, pp. 20-29.

[4] A. Catovic and Z. Sahinoglu, "The Cramér-Rao bounds of hybrid TOA/RSS and TDOA/RSS location estimation schemes," IEEE Commun. Lett., vol. 8, no. 10, pp. 626-628, Oct. 2004.

[5] E. G. Larsson, "Cramér-Rao bound analysis of distributed positioning in sensor networks," IEEE Signal Process. Lett., vol. 11, no. 3, pp. 334-337, March 2004.

[6] A. Savvides, W. Garber, R. Moses, and M. B. Srivastava, "An analysis of error inducing parameters in multihop sensor node localization," IEEE Transactions on Mobile Computing, vol. 4, no. 6, pp. 567-577, Nov./Dec. 2005.

[7] W. L. Stutzman and G. A. Theile, Antenna Theory and Design. John Wiley \& Sons, 1981.

[8] H. L. V. Trees, Detection, Estimation, and Modulation Theory, Part I. John Wiley \& Sons, 1968.

[9] S. M. Kay, Fundamentals of Statistical Signal Processing. New Jersey: Prentice Hall, 1993. 